Herz-Kreislauf-System

\section{Lebensqualität, Fitness und arterielle Steifigkeit nach Korrektur einer TGA}

Häcker AL et al. Functional outcomes in children with anatomically repaired transposition of the great arteries with regard to congenital ventricular septal defect and coronary pattern. Arch Dis Child 2019; 104: 851-856

Obwohl Mortalität und Morbidität zurückgehen, sind funktionelle Beeinträchtigungen nach operativer Korrektur einer Transposition der großen Arterien (TGA) weiterhin ein Problem, so die Autoren. So spielen neben Störungen der neurologischen Entwicklung motorische Defizite lebenslang eine große Rolle, ebenso eine reduzierte Elastizität der Arterien, eine Aortenwurzeldilatation, erhöhte Steifigkeit der Karotiden und eine Erhöhung des systolischen Blutdrucks und der Pulswellengeschwindigkeit.

Die vorliegende Studie analysiert die gesundheitsbezogene körperliche Fitness (HRPF, health-related physical fitness), arterielle Steifigkeit und gesundheitsbezogene Lebensqualität (HRQoL, healthrelated quality of life) bei Kindern mit anatomisch korrigierter TGA (arterielle Switch-Operation) auch unter Berücksichtigung eines kongenitalen Ventrikelseptumdefekts (VSD) und Lage und Funktion der Herzkranzarterien.

Von 2014-2017 wurden 68 Kinder mit anatomischer Korrektur einer TGA mit oder ohne VSD innerhalb eines Zentrums (München) untersucht. Das mittlere Lebensalter betrug 12,9 $\pm 3,7$ Jahre (Bandbreite 6-18 Jahre), 19,1\% waren Mädchen. Alle eingeschlossenen Patienten waren zum Untersuchungszeitpunkt (in Zusammenhang mit einem routinemäßigen Nachsorgetermin) in Stadium I-II der New York Heart Association-Klassifikation ohne Anzeichen einer Herzinsuffizienz, geistigen oder körperlichen Entwicklungsstörung, Syndromen oder Bewegungseinschränkungen. Aus den Patientenakten wurden krankheitsspezifische Daten übernommen, wie das Vorlie- gen eines VSD, eines atrialen Septumdefekts, eines offenen Foramen ovale, Prostaglandin-Gabe/Situation am Ductus Botalli, koronare Versorgung etc. Abhängig vom Vorliegen eines VSD wurden 2 Gruppen gebildet: 26 Patienten mit und 39 ohne VSD. Der VSD war dabei jeweils bei der chirurgischen Reposition der TGA mit verschlossen worden.

Die HRPF wurde durch 5 Tests in der FITNESSGRAM-Testbatterie bewertet. Die arterielle Steifigkeit wurde berechnet, nachdem oszillometrisch mit einem Mobil-o-Graphen Aortenpulswellengeschwindigkeit und zentraler systolischer Blutdruck gemessen wurden. Die HRQoL wurde durch einen Selbstbeurteilungsfragebogen (KINDL-R) erfasst. Alle Testergebnisse wurden mit einer gesunden Kontroll-Gruppe, adjustiert nach Lebensalter und Geschlecht, verglichen ( $\mathrm{n}=$ 2116, 49,1\% Mädchen).

Der zur Bestimmung der HRQoL verwendete KINDL-R-Fragebogen beinhaltet 24 Punkte, verteilt auf 6 Untergruppen, und bezieht sich auf die dem Test vorausgegangene Woche. Die 6 Untergruppen erfragen das körperliche Wohlbefinden, das seelische Wohlbefinden, das Selbstwertgefühl, Familie, Freunde und Alltagskompetenz. Geantwortet wird über eine 5-Punkt-Likert-Skala, die erreichbaren Punktwerte erstrecken sich von 0-100, der höchste Punktwert steht für die höchste HRQoL.

Kinder mit anatomischer Reposition der großen Gefäße hatten eine signifikant schlechtere HRPF (z-score: -0,58 $\pm 0,81$ vs. $0,02 \pm 0,63 ; p<0,001)$, eine erhöhte Pulswellengeschwindigkeit $(4,9 \pm 0,3 \mathrm{~m} / \mathrm{s}$ vs. $4,8 \pm 0,3 \mathrm{~m} / \mathrm{s} ; \mathrm{p}=0,028)$ und einen erhöhten zentralen systolischen Blutdruck $(105,9 \pm 5,8 \mathrm{mmHg}$ vs. 103,3 \pm $5,7 \mathrm{mmHg} ; \quad p=0,001)$, adjustiert nach Alter, Geschlecht, Body-Mass-Index, peripherem mittlerem arteriellem Druck, Medikation und Herzfrequenz. In der HRQoL konnte kein Unterschied zwischen den beiden Gruppen festgestellt werden (Gesamtpunktwert TGA: 76,5 \pm 10,2 vs. Kontroll-Gruppe: 75,2 $\pm 10,1$; $\mathrm{p}=0,315)$. Weder Charakteristika der Herzkranzarterien noch ein kongenitaler VSD bedeuteten einen signifikanten Un- terschied in allen funktionellen Ergebnissen.

Einschränkend geben die Autoren u.a. zu bedenken, dass durch die anatomische Korrektur der TGA die Pulmonalklappe anstelle der Aortenklappe verbleibt und die Retransposition der Aorta einen höheren und spitzeren Winkel des Aortenbogens nach sich zieht. Es ist noch nicht abschließend geklärt, inwieweit diese anatomischen Veränderungen zu einer veränderten Pulswellenreflexion führen, welche evtl. in einer falsch hohen Bestimmung der arteriellen Steifigkeit mündet.

FAZIT

Häcker et al. konnten bei Kindern mit anatomischer Korrektur einer TGA eine Beeinträchtigung der gesundheitsbezogenen Fitness sowie eine vermehrte arterielle Steifigkeit nachweisen. Die gesundheitsbezogene Lebensqualität unterschied sich jedoch nicht von der einer gesunden Kontroll-Gruppe. Die zugrundeliegenden koronaren Strukturen und/oder ein begleitender VSD scheinen keinen Einfluss auf das funktionelle Ergebnis zu haben.

\section{Dr. Birgit Gappa, Penzberg}

